\title{
Update After 4 Years of Use of the Improved Indwelling Intrauterine Tube (IIUT or 'Smit Sleeve') not Requiring Retaining Stitches for Well-Fractionated Brachy-Radiotherapy for Carcinoma of the Uterine Cervix
}

\author{
B J Smit ${ }^{1 *}$ and A L van Wijk ${ }^{2}$ \\ ${ }^{1}$ Emeritus Professor of Clinical Oncology, exTygerberg Academic Hospital, University of Stellenbosch, South Africa \\ ${ }^{2}$ Department of Radiation Oncology, Groote Schuur Hospital, University of Cape Town, South Africa
}

Received: 12 December, 2014; Accepted: 22 May, 2015; Published: 08 June, 2015

*Corresponding author: B J Smit, Emeritus Professor of Clinical Oncology, exTygerberg Academic Hospital, University of Stellenbosch, PO Box 63, Tygerberg 7505, South Africa, E-mail: bensmit@sonicwireless.co.za

\begin{abstract}
Objective: To report the experience after 4 years of use of the new version of the globally used 'Smit Sleeve' not requiring sutures as an ancillary device for well-fractionated, user-friendly and costeffective brachy-radiotherapy of carcinoma of the cervix of the uterus.

Methods: Injection moulding of suitable polymers of medical grade made many improvements to the original lathe-turned sleeve and injection moulded copies possible. The improved sleeves were used in 250 consecutive patients with carcinoma of the cervix. A problem with the old design was the need to stitch the sleeves in place and even then about $15 \%$ of them fell out and had to be repositioned.
\end{abstract}

Results: Not a single sleeve fell out in the 250 consecutive patients' representing1000 brachy-radiotherapy sessions. No complications related to the sleeves were observed.

Conclusions: The new sleeves were found to be superior to the older ones on all counts. By obviating stitching and loss, resterilization and re-insertion, they are cost- effective and more userfriendly to oncologist and patient alike and enable easy fractionation of dose.

Advances in knowledge: Improved manufacturing techniques improved an already useful device and rendered it safer, more hygienic, cost-effective and user friendly to patient and doctor alike.

\section{Objective}

The objective was to make a sleeve that would not require stitching to retain it properly in the uterus for periods of $1-3$ weeks at a time. The original, internationally used Indwelling Intra-Uterine Tube (IIUT) was first described in the British Journal of Radiology in 1989 [1,2]. This article showed that 4 to 16 fractions of High Dose Rate Remotely Controlled BrachyRadiotherapy (HDRBRT) were feasible with only one anaesthetic at initial insertion.

\section{Methods}

Two delicate wings added to the shaft of the sleeve would support ten times its own weight and would hold the sleeve either at the narrowing of the internal os or for very short sleeves, the external cervical os, thus preventing the sleeve from falling out. This removed the need for suturing the sleeve in place. The only way to achieve this design was by way of injection moulding with a medical grade polymer. This made possible introducing many additional features which were impossible before. The sleeves were made in different lengths from $30 \mathrm{~mm}$ to $80 \mathrm{~mm}$ automatically adjusting the isodose volumes to uterine size. Internal canal diameters of $3.7 \mathrm{~mm}, 4.0 \mathrm{~mm}$ and $4.5 \mathrm{~mm}$ made the sleeves suitable for $\mathrm{Ir}^{192}$ and, $\mathrm{Co}^{60}$ sources and for IMRT compatible polymer tandems with $4.0 \mathrm{~mm}$ outside diameter. The "wings" are very slender, flexible and soft projections 5.5 mm long x $2 \mathrm{~mm}$ wide $\mathrm{x} 1 \mathrm{~mm}$ thick with rounded, non-traumatic edges. Inert barium sulphate could be mixed in with the polymer granulate thus rendering the sleeves opaque to X-rays, CT and MRI and eliminated the need for metal markers. The sleeves could be made with thinner walls thus allowing lesser dilatation of the cervix ( $6.5 \mathrm{~mm}$ instead of $8 \mathrm{~mm}$ or more). The sleeves can be used either as disposable items or can be re-sterilized by chemical means. The mould also made provision for 'Universal sleeves' preferred by some oncologists. These sleeves are the same as the standard sleeves except that the tip is open so that any length of SGT/Tandem can be used. They are available in $40 \mathrm{~mm}, 50 \mathrm{~mm}$ and $70 \mathrm{~mm}$ lengths. It is essential that an inserter with a flange to push the stent in position is used. A suitable inserter has also been designed and made.

\section{Clinical Experience}

All aspects of the clinical utility of the original 'Smit Sleeve' have already been tested globally over a period of 20-plus years [1-4]. Proper fractionation of dose offers distinct radiobiological 
advantages $[5,6]$. Patients were fully informed of the nature of the treatment process and were required to sign consent. Between the two academic oncology departments in Cape Town circa 400 new patients with late stage cancer of the cervix are treated per annum and over a 20 year period more than 4000 patients have been treated.

We used the modified sleeves in 250 consecutive patients. Each sleeve stayed in position for 2-3 weeks while either a straight or angled Source Guide Tube (SGT) using either the Smit method [1,2] or with tandem-ring or tandem ovoid combinations inserted twice a week as per departmental protocol to deliver 4 fractions of High Dose Rate Brachy-Radiotherapy (HDRBRT) specified at "Point A" or equivalent.. During the resulting 1000 brachy-radiotherapy sessions, not a single sleeve of the new design fell out, nor was there any evidence of trauma, infection, discomfort or any untoward reaction. The sleeves were used exclusively initially over a period of 1 year from May 2010 till April 2011 and the results reported [7]. Today, the $20^{\text {th }}$ of November 2014 after having used the improved sleeves now for 53 months, only one sleeve has fallen out in a cervix destroyed by the carcinoma. If an older sleeve fell out, operating room time had to be rebooked and the sleeve had to be replaced. Since the new sleeves do not fall out, considerable time, trouble and costs are saved. Use of the cervical stent ('Smit Sleeve') is recommended for use by prospective users of Image Guided Brachytherapy (IGBT) for cervical carcinoma although for high incidence areas this method may dissuade oncologists from using brachytherapy especially in areas of high incidence [8].

\section{Conclusions}

The new sleeves are more user-friendly, cost-effective and safe and obviate the need for stitching. Other benefits apply to the operator, theatre staff, hospital and patient. None of the basic benefits of the original 'Smit Sleeves' were lost and other distinct advantages of the thinner, more flexible and versatile sleeves were demonstrated. The drawing (Figure 1) illustrates features of the new sleeve.

\section{Advances in Knowledge}

Re-thinking the design and changing the manufacturing process solved the significant problem of the need for suturing the sleeves and 'lost tube' problems were eliminated. Several other improvements were made possible due to the fact that tube walls could be made much thinner with injection moulding techniques. The delicate wings cannot be added except by injection moulding.

Figure 1 shows the modified Intra-uterine sleeve in position in the uterus. Note the delicate 'wings- -' sturdy enough to support about 5-10 times the weight of the sleeve. On insertion or extraction of the sleeve the soft wings simply fold away without

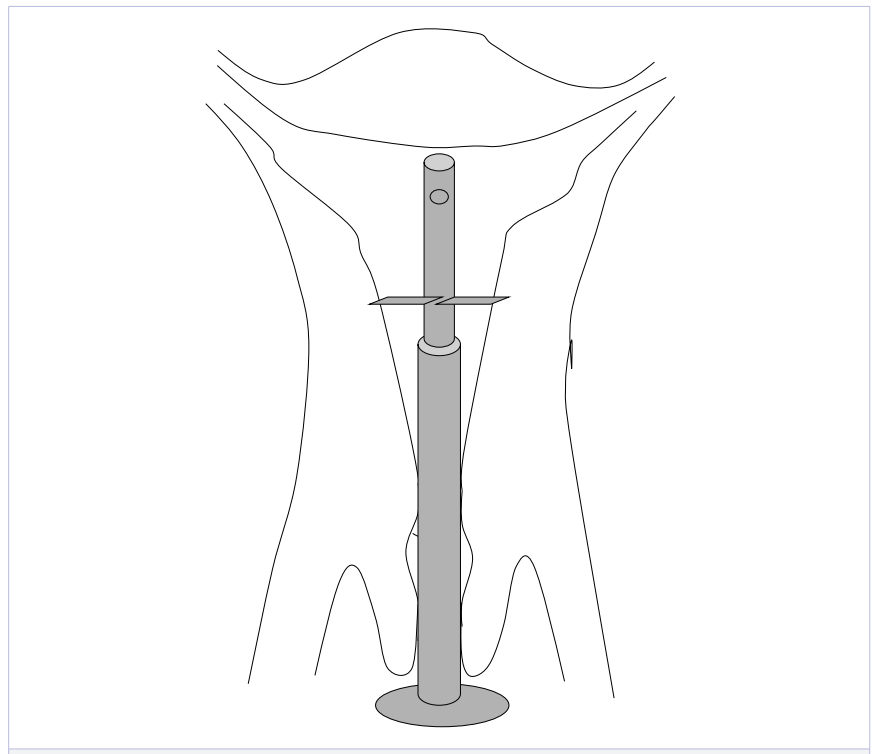

Figure 1: Modified Intra-uterine sleeve in position in the uterus.

causing trauma. The thin front end makes insertion easier. Dilatation of the cervix should be $0.5-1.0 \mathrm{~mm}$ more than the outside diameter of the thick section of the sleeve.

\section{References}

1. Smit BJ, du Toit JP, Groenewald WA. An Indwelling Intra-Uterine Tube to facilitate intra-cavitary radiotherapy of carcinoma of the cervix. $\mathrm{Br}$ J Radiol. 1989; 620 (733): 68-69.

2. Smit BJ. High Dose-Rate Brachy-radiotherapy for Carcinoma of the Cervix using the Indwelling Intra-Uterine Tube. Activity. 1991; 5: 2532.

3. Smit BJ. Design features of the Indwelling Intra-Uterine Tube for High Dose Rate Brachy-radiotherapy for carcinoma of the cervix and some hints on its optimal use. Br J Radiol. 1993; 66(791): 1042-1043.

4. du Toit JP, Smit BJ. Clinical prognostic factors in stage III cervical carcinoma - an analysis of 732 patients. S Afr Med J. 1997; 87(10): 1434-1440.

5. Smit BJ. Correspondence: One versus two or more Brachytherapy applications in cervical cancer. Int J Radiat Oncol Biol Phys. 1992; 23: 477.

6. Brenner DJ, Hall EJ. Fractionated, high dose rate versus low dose rate regimens for intra-cavitary brachytherapy of the cervix. Br J Radiol.1991; 64(758): 133-141.

7. Smit BJ, van Wijk AL. An improved disposable indwelling intra-uterine tube ("Smit Sleeve") not requiring retaining stitches for brachyradiotherapy of carcinoma of the cervix. Eur J Gynaecol Oncol. 2013; 34(4): 289-290.

8. Implementing image guided brachytherapy for cervix cancer in the UK. The Royal College of Radiologists. 2009; London, UK. 